\title{
Coherent large-scale structures from the linearized Navier-Stokes equations
}

\author{
Anagha Madhusudanan ${ }^{1} \dagger$, Simon. J. Illingworth ${ }^{1}$ and Ivan Marusic ${ }^{1}$ \\ ${ }^{1}$ Department of Mechanical Engineering, University of Melbourne, Melbourne, VIC 3010, \\ Australia \\ (Received $\mathrm{xx}$; revised $\mathrm{xx}$; accepted $\mathrm{xx}$ )
}

The wall-normal extent of the large-scale structures modelled by the linearized NavierStokes equations subject to stochastic forcing is directly compared to Direct Numerical Simulation (DNS) data. A turbulent channel flow at a friction Reynolds number of $R e_{\tau}=$ 2000 is considered. We use the two-dimensional (2-D) linear coherence spectrum (LCS) to perform the comparison over a wide range of energy-carrying streamwise and spanwise length scales. The study of the 2-D LCS from DNS indicates the presence of large-scale structures that are coherent over large wall-normal distances and that are self-similar. We find that, with the addition of an eddy viscosity profile, these features of the largescale structures are captured by the linearized equations, except in the region close to the wall. To further study this coherence, a coherence-based estimation technique, spectral linear stochastic estimation (SLSE), is used to build linear estimators from the linearized Navier-Stokes equations. The estimator uses the instantaneous streamwise velocity field or the 2-D streamwise energy spectrum at one wall-normal location (obtained from DNS), to predict the same quantity at a different wall-normal location. We find that the addition of an eddy viscosity profile significantly improves the estimation.

\section{Introduction}

Large-scale coherent structures in the outer layer of wall-bounded turbulent flows play a crucial role in these flows. The smaller among these structures, known as LSMs, have a characteristic length scale of two to three times the boundary layer thickness (Brown \& Thomas 1977; Kim \& Adrian 1999; Zhou et al. 1999; Adrian et al. 2000; Ganapathisubramani et al. 2003; Hutchins et al. 2005; Tomkins \& Adrian 2005; Dennis \& Nickels 2011; Jiménez 2012). The largest structures, often referred to as very largescale motions (VLSMs) or superstructures, are very long regions of low momentum that meander in the streamwise direction. They can extend for 10 to 15 times the boundary layer thickness, or up to 30 times the channel half-height in internal flows (Kim \& Adrian 1999; Adrian et al. 2000; Tomkins \& Adrian 2005; Guala et al. 2006; Balakumar \& Adrian 2007; Hutchins \& Marusic 2007b,a; Monty et al. 2007; Smits et al. 2011). Geometrically self-similar eddies of the type proposed by Townsend in his attached eddy hypothesis (AEH) (Townsend 1976) have been used to conceptually describe these structures (e.g. Marusic (2001); Klewicki et al. (2009); Lozano-Durán et al. (2012); Hwang (2015); Hellström et al. (2016)).

Within the logarithmic layer of flows at high Reynolds numbers, the LSMs and the VLSMs contribute significantly to the turbulent kinetic energy and the Reynolds shear stress (Tomkins \& Adrian 2005; Guala et al. 2006; Balakumar \& Adrian 2007). Consequently their modelling is important both for practical flow control purposes and

$\dagger$ Email address for correspondence: amadhusudana@student.unimelb.edu.au 
for furthering the theoretical understanding of these flows. To model and estimate these large-scale structures using experimental data, stochastic estimation techniques and the linear coherence spectrum (LCS) have been useful. Stochastic estimation is used for the estimation of turbulent flows (Adrian 1979; Adrian \& Moin 1988; Cole \& Glauser 1998; Bonnet et al. 1998), where given the measurement of the velocity signal at a point in space and in time, an estimated velocity signal is obtained at another point in space. This method was later extended into Spectral Linear Stochastic Estimation (SLSE), where the estimation is performed in Fourier space (Ewing \& Citriniti 1999; Tinney et al. 2006). For SLSE, it is necessary to isolate the coherent scales that can be estimated. For this purpose, the Linear Coherence Spectrum (LCS) was defined (Tinney et al. 2006), which gives the fraction of energy that is correlated between two signals. Later, Baars et al. (2017) observed the self-similar scaling of the LCS computed from turbulent channel and boundary layer flows. This observed scaling gives further support for Townsend's attached eddy hypothesis (Townsend 1976; Marusic \& Monty 2019).

As well as experimental efforts to understand the coherent large-scale structures, their modelling has also received attention. Many of these efforts investigated whether the nonnormality of the Navier-Stokes equations linearized around the turbulent mean velocity profile can explain the large-scale coherent structures that have been observed in fully turbulent flows. This built on earlier work that showed the non-normality of the NavierStokes equations linearized around the laminar velocity profile gives rise to streamwise streaks (Trefethen et al. 1993; Schmid 2007). To understand the structures modelled by the Navier-Stokes equations linearized around the turbulent mean velocity profile, the perturbations that experience the maximum transient growth due to the non-normality of the equations have been studied (Butler \& Farrell 1993; Farrell \& Ioannou 1993), and the sensitivity of the equations to initial perturbations has been analysed (Farrell \& Ioannou 1998). From these studies, it was observed that the structures that are most amplified by the model are coherent streaks elongated in the streamwise direction that are reminiscent of the streaks observed in experiments. However, the spanwise dimensions of the streaks in the near-wall region do not match the spanwise spacing of the near-wall streaks found in experiments (Waleffe et al. 1993; Butler \& Farrell 1993).

By following Reynolds \& Hussain (1972) and augmenting the kinematic viscosity with an eddy viscosity that varies with wall height, the spanwise dimensions of the streamwise streaks show closer agreement to the values from experiments (Del Álamo \& Jiménez 2006; Pujals et al. 2009; Cossu et al. 2009; Hwang \& Cossu 2010a,b; Willis et al. 2010). This eddy viscosity based model can also approximately estimate the large-scale features of a turbulent channel flow at $R e_{\tau}=1000$ (Illingworth et al. 2018). Recent work has used a resolvent framework to show that some key features of these coherent large-scale structures can also be captured without the use of an eddy viscosity profile (McKeon \& Sharma 2010; McKeon et al. 2013; Sharma \& McKeon 2013; Moarref et al. 2013, 2014). In this framework, the linearized Navier-Stokes equations are forced by the remaining nonlinear terms.

In the current work, we study the wall-normal coherence of the large-scale structures modelled by the linearized Navier-Stokes equations, over a range of energy-carrying length scales. The study of wall-normal coherence is important because it gives us an understanding of the wall-normal extent of the structures as a function of their streamwise and spanwise length scales. The LCS that has been used in experiments is used here to quantify the wall-normal coherence of the structures. The quantification of wall-normal coherence enables a direct comparison of the wall-normal extent of the large-scale structures from the model with DNS. To further study the coherent large-scale structures, we use a coherence based estimation technique called spectral linear stochastic 
estimation (SLSE) (Tinney et al. 2006; Baars et al. 2016) to build linear estimators using the linearized Navier-Stokes equations. The estimators take as input the instantaneous velocity field or the 2-D energy spectrum at one wall-normal location to provide the estimate of the same quantity at a different wall-normal location.

The nonlinear terms of the linearized equations are considered to act as a forcing, as done in McKeon \& Sharma (2010). Additionally, this forcing is assumed to be stochastic and white-in-time (Hwang \& Cossu $2010 a, b$; Willis et al. 2010). Following the discussion above, two variations of the model are considered: i) LNS (linearized Navier-Stokes equations), where the viscosity is equal to the kinematic viscosity and ii) eLNS (eddyviscosity based linearized Navier-Stokes equations), where the kinematic viscosity is augmented with an eddy viscosity profile. These models are described in $\S 2$. The details of the DNS dataset obtained from Encinar et al. (2018), that are used for comparison are given in $\S 3$. We compare LNS and eLNS with DNS based on two aspects: i) the coherence of the large-scale structures in $\S 4$ and ii) the estimates of the instantaneous streamwise velocity and the 2-D streamwise energy spectrum obtained using SLSE in $\S 5$. In both of these comparisons, we will see that the results are significantly improved by the inclusion of an eddy viscosity profile.

\section{Linear model}

A statistically steady, incompressible turbulent channel flow is considered, with the streamwise, spanwise and wall-normal directions denoted by $x, y$ and $z$, respectively, and the corresponding velocity components by $u, v$ and $w$. The Reynolds number is $R e_{\tau}=2000$. Here, the friction Reynolds number $R e_{\tau}=u_{\tau} h / \nu$ is defined using the kinematic viscosity $\nu$, the channel half-height $h$ and the friction velocity $u_{\tau}=\sqrt{\left(\tau_{w} / \rho\right)}$, where $\tau_{w}$ is the wall shear stress and $\rho$ is the density. The velocities are normalized by $u_{\tau}$ and the spatial variables by $h$. The non-dimensional channel half-height then becomes unity. In this paper a ' + ' superscript indicates the normalization of the spatial variables by the viscous length scale $\nu / u_{\tau}$. The pressure fluctuations $p$ are normalized by $\rho u_{\tau}^{2}$.

Before describing the linear models used, we first introduce the Cess (1958) eddy viscosity profile which will be used in different ways for both the models described below. The Cess (1958) eddy viscosity model defines a total viscosity $\nu_{T}(z)$ as the sum of a constant molecular viscosity and an eddy viscosity that varies in the wall-normal direction. As a function of $z$, this total viscosity profile is

$$
\nu_{T}(z)=\frac{\nu}{2}\left(1+\frac{\kappa^{2} R e_{\tau}^{2}}{9}\left(2 z-z^{2}\right)^{2}\left(3-4 z+z^{2}\right)^{2}\left[1-\exp \left(\frac{-R e_{\tau} z}{A}\right)\right]^{2}\right)^{1 / 2}+\frac{\nu}{2} .
$$

For convenience, here the mean velocity profile of the flow is obtained from (2.1) by integrating the expression $\operatorname{Re}_{\tau}(1-z) \nu / \nu_{T}$ in the wall-normal direction (Reynolds \& Tiederman 1967). The values of the constants in (2.1) are taken to be $\kappa=0.426$ and $A=25.4$, following Del Álamo \& Jiménez (2006), where they were obtained through a least-square fit to experimentally obtained mean velocity profiles at $R e_{\tau}=2000$.

\section{1. $L N S$}

The first linear model is obtained by first substituting a Reynolds decomposition into the Navier-Stokes equations and then subtracting the mean equations, which gives

$$
\frac{\partial \boldsymbol{u}}{\partial t}+(\boldsymbol{U} . \nabla) \boldsymbol{u}+(\boldsymbol{u} . \nabla) \boldsymbol{U}+\nabla \boldsymbol{p}-\frac{1}{R e_{\tau}} \nabla^{2} \boldsymbol{u}=\boldsymbol{d}_{L N S}, \quad \nabla . \boldsymbol{u}=0,
$$


where $\boldsymbol{U}=(U(z), 0,0)$ is the mean velocity profile that is obtained using (2.1) and $\boldsymbol{u}=(u, v, w)$ denotes the fluctuations of velocity from the mean. Following McKeon \& Sharma (2010), the nonlinear terms are represented by a disturbance term $\boldsymbol{d}_{L N S}=$ $-\boldsymbol{u} . \nabla \boldsymbol{u}+\overline{\boldsymbol{u} . \nabla \boldsymbol{u}}$. Here, $\boldsymbol{d}_{L N S}$ is assumed to be stochastic and white-in-time (Hwang \& Cossu 2010a).

\section{2. $e L N S$}

The second linear model is obtained by substituting into the Navier-Stokes equations a triple decomposition of the velocity field as $\tilde{\boldsymbol{u}}=\boldsymbol{U}+\boldsymbol{u}+\boldsymbol{u}^{\prime}$, where $\boldsymbol{U}$ as before is the mean velocity, the term $\boldsymbol{u}$ denotes the organised motions and $\boldsymbol{u}^{\prime}$ represents the turbulent velocity fluctuations (Reynolds \& Hussain 1972; Del Álamo \& Jiménez 2006; Pujals et al. 2009; Hwang \& Cossu 2010b). The Cess (1958) eddy viscosity profile (2.1) is used to model the terms that are quadratic in $\boldsymbol{u}^{\prime}$. This model can be written as

$$
\frac{\partial \boldsymbol{u}}{\partial t}+(\boldsymbol{U} . \nabla) \boldsymbol{u}+(\boldsymbol{u} . \nabla) \boldsymbol{U}+\nabla \boldsymbol{p}-\nabla \cdot\left[\frac{\nu_{T}(z)}{\nu}\left(\nabla \boldsymbol{u}+\nabla \boldsymbol{u}^{T}\right)\right]=\boldsymbol{d}_{e L N S}, \quad \nabla \cdot \boldsymbol{u}=0 .
$$

The mean velocity profile is again obtained using (2.1). Additionally, (2.1) also provides the eddy viscosity profile $\nu_{T}(z)$ required for (2.3). The term, $\boldsymbol{d}_{e L N S}$ represents the forcing, but defined using the $\boldsymbol{u}$ obtained from the triple decomposition of the velocity field. As before, $\boldsymbol{d}_{e L N S}$ is assumed to be stochastic and white-in-time.

\subsection{Orr-Sommerfeld Squire form}

The models in (2.2) and (2.3) can now be written in the Orr-Sommerfeld Squire form. A 2-D Fourier transformation of $\boldsymbol{u}$ and $\boldsymbol{d}$ along the homogeneous streamwise and spanwise directions gives the respective Fourier coefficients $\hat{\boldsymbol{u}}\left(z, t ; k_{x}, k_{y}\right)=(\hat{u}, \hat{v}, \hat{w})$ and $\hat{\boldsymbol{d}}\left(z, t ; k_{x}, k_{y}\right)=\left(\hat{d}_{x}, \hat{d}_{y}, \hat{d}_{z}\right)$. Here $\left(k_{x}, k_{y}\right)$ are the streamwise and spanwise wavenumbers, and $\left(\lambda_{x}, \lambda_{y}\right)$ the corresponding wavelengths. In terms of these Fourier coefficients, the Orr-Sommerfeld Squire form of the models (2.2) and (2.3) is (Del Álamo \& Jiménez 2006; Hwang \& Cossu 2010b)

$$
\begin{aligned}
& \dot{\hat{\boldsymbol{q}}}=\boldsymbol{A} \hat{\boldsymbol{q}}+\boldsymbol{B} \hat{\boldsymbol{d}}, \\
& \hat{\boldsymbol{u}}=\boldsymbol{C} \hat{\boldsymbol{q}},
\end{aligned}
$$

where the definitions of the matrices $\boldsymbol{A}, \boldsymbol{B}$ and $\boldsymbol{C}$ for both LNS and eLNS are given in appendix A. The vector $\hat{\boldsymbol{q}}=(\hat{w}, \hat{\eta})$ is formed with Fourier coefficients of the wall-normal velocity and wall-normal vorticity. The boundary conditions are enforced on both walls as $\hat{w}( \pm h)=\partial \hat{w}( \pm h) / \partial z=\hat{\eta}( \pm h)=0$. A Chebyshev grid with 203 grid points is used to discretize the above equations in the wall-normal direction and the convergence of the results is ensured by reproducing them with more than double the number of grid points.

\subsection{Lyapunov equations}

Time-averaged velocity correlations from LNS and eLNS are required in $\S 4$ to compute the linear coherence spectrum (LCS), that will be used to understand the coherence of the structures from the models. These correlations required for the LCS can be obtained using Lyapunov equations. From the equations written in the Orr-Sommerfeld Squire form (2.4), the Lyapunov equations are derived as (Zhou et al. 1996)

$$
\boldsymbol{A}\left(k_{x}, k_{y}\right) \boldsymbol{X}\left(k_{x}, k_{y}\right)+\boldsymbol{X}\left(k_{x}, k_{y}\right) \boldsymbol{A}\left(k_{x}, k_{y}\right)^{*}=-\boldsymbol{B}\left(k_{x}, k_{y}\right) \boldsymbol{B}\left(k_{x}, k_{y}\right)^{*} .
$$

Here the matrix $\boldsymbol{X}$ gives the correlations of the velocity-vorticity vector $\left\langle\hat{\boldsymbol{q}} \hat{\boldsymbol{q}}^{H}\right\rangle$, and the

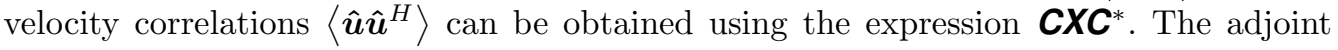


* here is defined with respect to the inner product $\langle\boldsymbol{u} \mathbf{1}, \boldsymbol{u} \mathbf{2}\rangle=\int_{-h}^{h} \boldsymbol{u} \mathbf{1}^{H} \boldsymbol{u} \mathbf{2} d z$. The Lyapunov equation (2.5) can be solved both for LNS and eLNS giving the velocity correlation matrices for these models as its solution.

\section{DNS Dataset}

The Direct Numerical Simulation (DNS) dataset for an incompressible turbulent channel flow at a friction Reynolds number $R e_{\tau}=2000$ has been provided by the Polytechnic University of Madrid (UPM) (Encinar et al. 2018). The channel has a streamwise and spanwise extent of $8 \pi h$ and $3 \pi h$. The DNS was run on a grid with $2048 \times 2048 \times 512$ points in the streamwise, spanwise and wall-normal directions. By retaining only the scales that are larger than the viscous scales, Encinar et al. (2018) stored the data on a reduced grid of size $512 \times 512 \times 512$. Only a subset of the saved wavenumbers are available for this study, and the range of wavenumbers available are $0.25 \leqslant\left|k_{x} h\right| \leqslant 8.0 \quad\left(0.8 \leqslant\left|\lambda_{x} / h\right| \leqslant 25.0\right)$ and $0.66 \leqslant\left|k_{y} h\right| \leqslant 21.0 \quad\left(0.3 \leqslant\left|\lambda_{y} / h\right| \leqslant\right.$ 9.5). This range includes the large-scale structures that are of interest here. It has been confirmed that the 1146 instances in time for which data are available gives rise to a converged 2-D energy spectrum by comparing the spectra that was computed with the converged spectra available from Hoyas \& Jiménez (2006).

\section{Coherent large-scale structures from the linear models}

We now investigate the coherence of the large scales using the linear coherence spectrum, coherence height and the scaling of the linear coherence spectrum.

\subsection{Linear Coherence Spectrum}

The linear coherence spectrum gives the fraction of energy that is correlated between two signals (Tinney et al. 2006). In the present case, these signals correspond to streamwise velocity. The LCS has been used to study the coherence between velocity signals that were obtained as a function of one dimension; the dimension of time in Tinney et al. (2006) and of streamwise length in Baars et al. (2016). In Baars et al. (2016) the 1-D LCS was defined for turbulent channel and boundary layer flows, between signals taken at two wall-normal locations $z_{1}$ and $z_{2}$. Here, the 1-D LCS in Baars et al. (2016) is extended to also include the spanwise variation in the velocity signals, thereby obtaining a 2-D LCS as a function of both the streamwise and spanwise wavenumbers $k_{x}$ and $k_{y}$. The 2-D LCS, denoted here by $\gamma^{2}$, can be written as

$$
\gamma^{2}\left(z_{1}, z_{2} ; k_{x}, k_{y}\right)=\frac{\left|\left\langle\hat{u}\left(z_{1} ; k_{x}, k_{y}\right) \hat{u}^{*}\left(z_{2} ; k_{x}, k_{y}\right)\right\rangle\right|^{2}}{\left\langle\left|\hat{u}\left(z_{1} ; k_{x}, k_{y}\right)\right|^{2}\right\rangle\left\langle\left|\hat{u}\left(z_{2} ; k_{x}, k_{y}\right)\right|^{2}\right\rangle} .
$$

Here, as in $\S 2, \hat{u}\left(z ; k_{x}, k_{y}\right)$ represents the coefficients of a 2-D Fourier transform of the streamwise velocity signal $u$ at a wall height $z$.

The denominator in (4.6) consists of two individual 2-D energy spectra at the wall heights $z_{1}$ and $z_{2}$, while the numerator is the absolute value of the complex valued crossspectrum between the two wall heights. By definition, $0 \leqslant \gamma^{2} \leqslant 1$, where $\gamma^{2}=1$ indicates perfect coherence and $\gamma^{2}=0$ indicates no coherence. Before using (4.6) to analyse the linear models, we first plot in figure 1(b) an example of the LCS using the DNS data described in $\S 3$. Also shown in figure 1(a) is a schematic of the geometry for which the calculations are performed. We are interested in studying the coherent large-scale structures in the log-layer of the flow. Hence, $z_{2}^{+} \approx 300\left(=0.15 R e_{\tau}\right)$ is kept fixed at the 

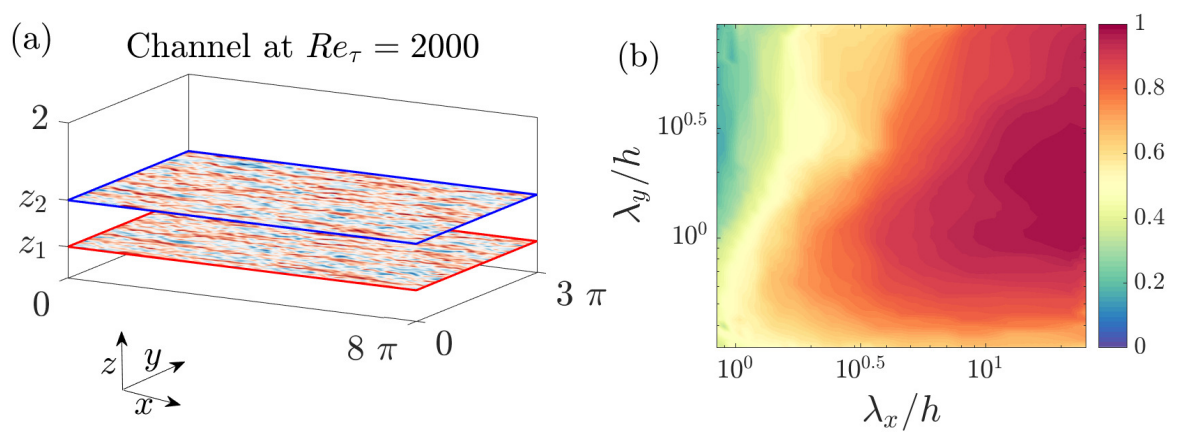

Figure 1: (a) The geometry of the turbulent channel flow. (b) The LCS plotted for $z_{1}$ and $z_{2}$ as depicted in (a), with $z_{2}^{+} \approx 300\left(=0.15 R e_{\tau}\right)$ kept at the end of the log-layer and $z_{1}^{+} \approx 200$.

end of the log-layer (Marusic et al. 2013), and $z_{1}$ is taken beneath it. For the example in figure $1(\mathrm{~b}), z_{1}^{+} \approx 200$ and the 2-D LCS is plotted as a function of $\lambda_{x} / h$ and $\lambda_{y} / h$. We observe that the larger scales are more coherent than the smaller scales.

As well as plotting the LCS for the DNS data, we can also compute it for the linear models (2.2) and (2.3). In this case, rather than using velocity signals directly, the statistically converged velocity correlations required for (4.6) are obtained by solving the Lyapunov equation (2.5). To obtain the solution, (2.2) and (2.3) are discretized in the wall-normal direction using $N_{z}=203$ Chebyshev points (see $\S 2$ ). The solution to (2.5) gives the velocity correlations $\left|\left\langle\hat{\boldsymbol{u}}\left(z_{1} ; k_{x}, k_{y}\right) \hat{\boldsymbol{u}}^{*}\left(z_{2} ; k_{x}, k_{y}\right)\right\rangle\right|$ for any combination of wall-heights $z_{1}$ and $z_{2}$, and therefore provides the correlations required for (4.6). Figure 2 shows the LCS computed for DNS, LNS and eLNS for five different values of $z_{1}$.

Let us first consider the LCS from DNS. We start with the example shown in figure 1 , which is shown again in figure 2 (a). Here the first wall height, $z_{1}^{+} \approx 200$ is relatively close to the second wall height, $z_{2}^{+} \approx 300$, which remains fixed throughout. Moving down the rows in figure 2 (from (a) to (e)) corresponds to moving the first wall height $z_{1}$ closer to the wall and further away from $z_{2}$. For every pair of wall heights we see that the larger scales are more coherent than the smaller scales. Interestingly, the coherence of the largest scales remains high even when $z_{1}$ is moved very close to the wall (figure $2(\mathrm{e})$ ). From this we can infer the existence of structures that are attached to the wall and that extend to the end of the log-layer, consistent with the observations from the 1-D LCS in Baars et al. (2017).

Now consider the LCS computed from LNS. From figure 2(a) it can be seen that, like in DNS, the large scales are more coherent than the smaller scales when $z_{1}$ and $z_{2}$ are close to each other. However, the contours from LNS in figure 2(a) look notably different to those computed from DNS. We also see that, unlike in DNS, the coherence of the large scales quickly approaches zero as $z_{1}$ moves away from $z_{2}$ and towards the wall. This indicates that the structures modelled by LNS are localised in the wall-normal direction.

Finally, we examine the LCS computed from eLNS. Figure 2(a) shows that the large scales from this model also show a higher degree of coherence than the smaller scales. The contours from eLNS look similar to DNS. As $z_{1}$ moves away from $z_{2}$ (from (a) to (d)), the coherence of the large scales from eLNS remains high and the contours remain similar to DNS. However, when $z_{1}$ is moved close to the wall (figure 2(e)), the correspondence with DNS is lost and the large scales from the model show diminished coherence. Hence 
the structures from eLNS are coherent over a wider range of wall-heights, but they show lower coherence than DNS in the region close to the wall.

\subsection{Coherence Height}

In $\S 4.1$ the coherence of the large-scale structures was studied as a function of two wall-normal locations $z_{1}$ and $z_{2}$. The coherence of the large scales from eLNS was found to be in agreement with DNS except in the near-wall region. However, it is unclear if these results are specific to the particular choice of $z_{2}^{+} \approx 300$ considered in $\S 4.1$. Hence, to probe into the agreement between DNS and eLNS over a range of $z_{1}$ and $z_{2}$ combinations, the concept of a coherence height, as defined in Jiménez et al. (2004), is now used. Since the coherence of the large scales from LNS was found to disagree with DNS, this model is not considered here. The coherence height $C_{u u}$ is defined using $\gamma$ (from (4.6)) as

$$
C_{u u}\left(z_{0} ; k_{x}, k_{y}\right)=\left(\int_{0}^{z_{0}} \int_{0}^{z_{0}} \gamma d z_{1} d z_{2}\right)^{1 / 2} .
$$

Since $\gamma$ is a dimensionless quantity, $C_{u u}$ has the dimensions of length. Coherence height gives the approximate height over which a structure is coherent. The first integral in (4.7) is equivalent to calculating $\gamma$ while keeping one probe fixed at $z_{2}$ and varying the second probe over all $z_{1}$, such that $0 \leqslant z_{1} \leqslant z_{0}$. This returns a 'height' that depends on the choices of $z_{0}$ and also $z_{2}$. The second integral calculates this height for all positions of the second probe $z_{2}$ such that $0 \leqslant z_{2} \leqslant z_{0}$ and hence returns a squared height that depends only on $z_{0}$.

The integral in (4.7) can be calculated for different values of the integration limit $z_{0}$. The value of $z_{0}$ sets the region of the channel being considered. For a wavenumber pair $\left(k_{x}, k_{y}\right), C_{u u}\left(z_{0}, k_{x}, k_{y}\right)$ considers the coherence between all combinations of wall heights $z_{1}$ and $z_{2}$ such that $0 \leqslant z_{1} \leqslant z_{0}$ and $0 \leqslant z_{2} \leqslant z_{0}$. Figure 3 plots the coherence height as a function of $\lambda_{x} / h$ and $\lambda_{y} / h$ from DNS and eLNS, for two values of $z_{0}^{+} ; \mathrm{i}$ ) $z_{0}^{+} \approx 300\left(=0.15 R e_{\tau}\right)$ in figure $3(\mathrm{a})$ and ii) $z_{0}^{+} \approx 40$ in figure $3(\mathrm{~b})$.

First consider figure $3(\mathrm{a})$ where the coherence height is computed with $z_{0}^{+} \approx 300$. The large-scale structures from DNS have coherence heights very close to the integration limit $z_{0}$. This indicates that these structures extend across most of the region between the wall and $z_{0}^{+} \approx 300$. Considering eLNS, the contours from this model in figure 3(a) approximately correspond to those from DNS, with the large scales having coherence heights close to $z_{0}$. Hence the large-scale structures modelled by eLNS are also coherent across most of the region till the end of the log-layer.

Now consider figure $3(\mathrm{~b})$ where the integration limit is taken at $z_{0}^{+} \approx 40$. We see that even in this case, the large-scale structures from DNS show coherence heights very close to $z_{0}$, suggesting that these structures extend all the way to the wall. However, the contours from eLNS in this figure deviate in their trends from DNS, indicating that the features of the flow in the near-wall region are not captured by eLNS. Therefore, coherence of the large-scale structures are modelled reasonably well by eLNS, except in the region close to the wall. These observations are consistent with those from the LCS in $\S 4.1$. 

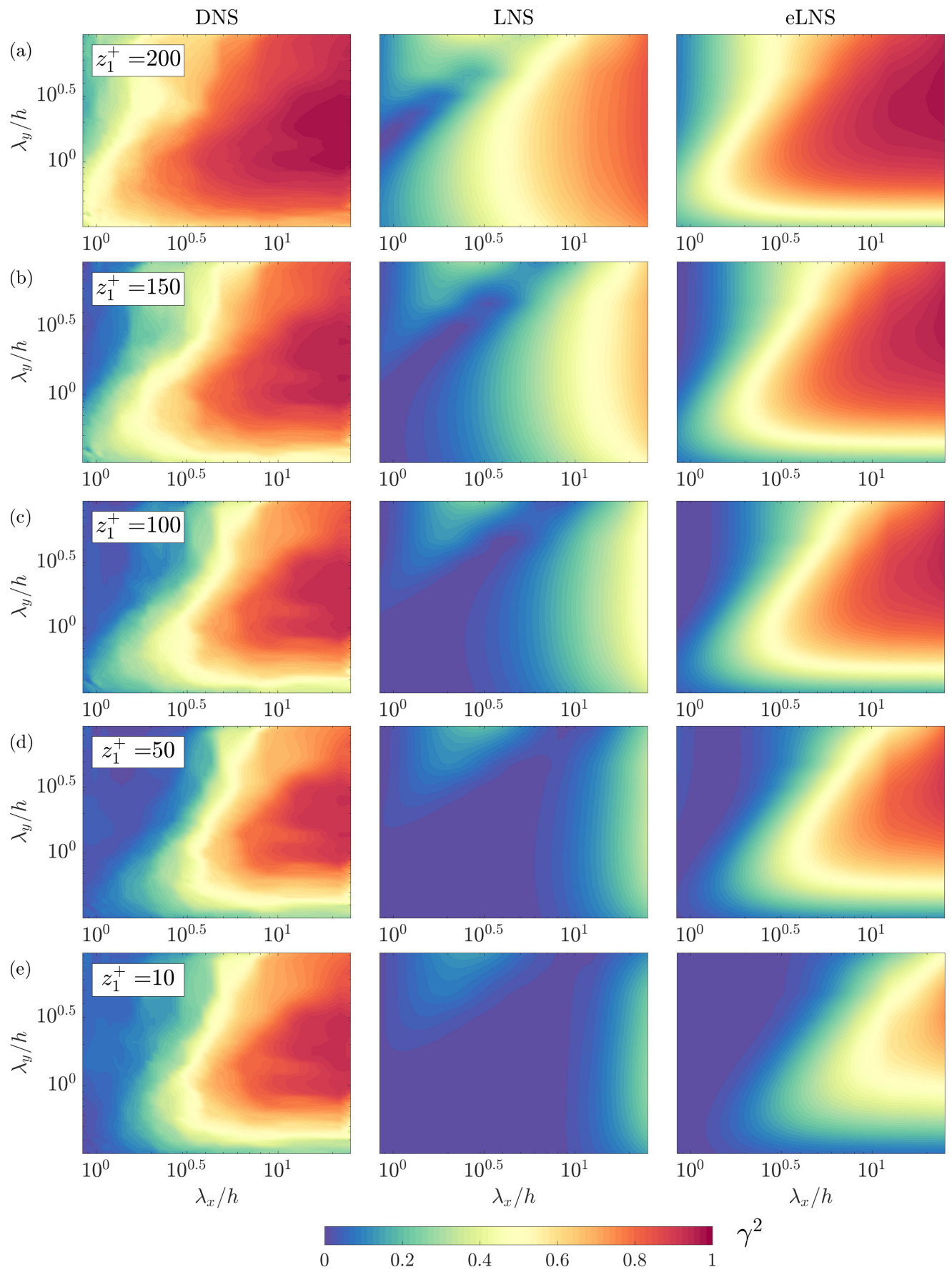

Figure 2: The LCS plotted from LNS and eLNS and compared with the LCS from DNS, with $z_{2}^{+} \approx 300\left(=0.15 R e_{\tau}\right)$, and $z_{1}^{+}$varied beneath $z_{2}^{+}$. The plots corresponding to $z_{1}^{+} \approx 200,150,100,50$ and 10 are shown from the top row to the bottom row. 

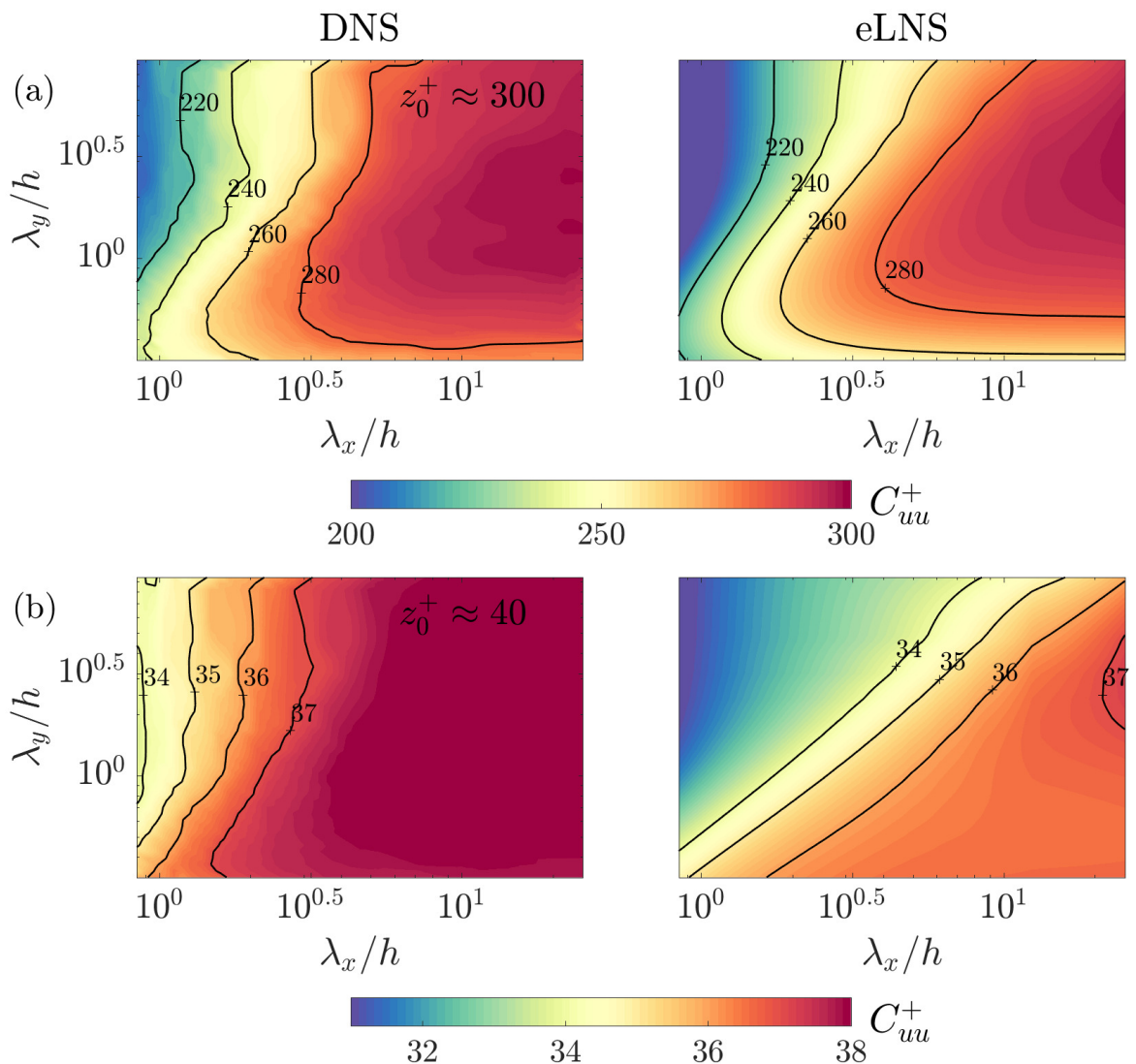

Figure 3: Coherence height computed from DNS and eLNS with (a) $z_{0}^{+} \approx 300$ and (b) $z_{0}^{+} \approx 40$. The line contours in (a) corresponds to $C_{u u}^{+} \approx(220,240,260,280)$ and in (b) to $C_{u u}^{+} \approx(34,35,36,37)$.

\subsection{Scaling of the coherence spectrum}

Having observed the presence of large-scale structures in eLNS that are similar to those from DNS, it is important to identify if the structures from the model are selfsimilar. Many authors have found evidence for the self-similar scaling of these large-scale structures and hence for Townsend's attached eddy hypothesis (AEH) in turbulent flows (e.g. Marusic (2001); Klewicki et al. (2009); Lozano-Durán et al. (2012); Hwang (2015); Hellström et al. (2016). The linearized Navier-Stokes equations have also been used to understand this geometric self-similarity (Del Álamo \& Jiménez 2006; McKeon \& Sharma 2010; Hwang \& Cossu 2010b; Moarref et al. 2013).

Of particular relevance to this study is the coherence-based analysis carried out by Baars et al. (2017) to demonstrate this self-similar scaling of the large-scale structures. For this purpose, Baars et al. (2017) used the wall scaling of the experimentally obtained 1-D LCS plotted as a function of $\lambda_{x}$. Wall scaling implies that the 1-D LCS scales with $z_{2}$, when plotted for a range of $z_{2}$ in the log-layer and $z_{1}$ fixed close to the wall. Since $\lambda_{x}$ represents the streamwise length of the structures, wall scaling implies the existence of self-similar structures. The streamwise lengths of the structures scale with their height, in accordance with AEH. 
Due to self-similarity, the spanwise dimensions of the structures should also scale with their height. The arguments in Baars et al. (2017) can therefore be extended to two dimensions, and the contours of 2-D $\gamma^{2}$ should scale with $z_{2}$ for a range of $z_{2}$. For this argument, $z_{1}$ is taken close to the wall at $z_{1}^{+} \approx 40$ and $z_{2}$ is varied within the loglayer, and hence $2.6 \sqrt{R e_{\tau}} \leqslant z_{2}^{+} \leqslant 0.15 R e_{\tau}$ (Klewicki et al. 2009; Marusic et al. 2013). (Here $z_{1}$ is not as close to the wall as in Baars et al. (2017), where $z_{1}^{+} \approx 4$. This is to facilitate a comparison with eLNS where, as observed in $\S 4.1$, the large scales show very low coherence close to the wall.)

First, we verify the scaling of the 2-D $\gamma^{2}$ using the DNS dataset. Figure 4(a) shows the contours corresponding to $\gamma^{2}=0.3$ as a function of $\left(\lambda_{x} / h, \lambda_{y} / h\right)$ for a range of $z_{2}$. Each line contour in the figure is plotted for one value $z_{2}$ in the range considered. The contours collapse when plotted as a function of the wavelengths scaled with $z_{2}$ in figure 4(b). Figure 4(b) also show the collapse of the contours corresponding to $\gamma^{2}=0.1$ and $\gamma^{2}=0.5$. Therefore, the 2-D LCS from DNS shows wall scaling and thereby indicates the presence of self-similar structures in the flow.

Now we consider the scaling of the LCS from the linear models. The LCS from LNS do not show wall scaling. In contrast, the LCS from eLNS does scale with wall height, and therefore only the results from this model are discussed here. Figures 4(c) and 4(d) demonstrate the wall scaling of the LCS from eLNS by re-plotting figures 4(a) and 4(b) respectively, for the model. In figure $4(\mathrm{~d})$ we observe the collapse of the contours when plotted as a function of $\left(\lambda_{x} / z_{2}, \lambda_{y} / z_{2}\right)$. The contours collapse for approximately the same range of scales as in DNS. This self-similar scaling of the 2-D LCS from eLNS indicates that the model not only captures the coherence of the large-scale structures of the flow, but also captures their self-similar behaviour.

\subsection{A discussion of the coherent large-scale structures from the linear models}

The 2-D LCS reveals that LNS gives rise to structures that are localised in the wallnormal direction (figure 2). This is indicative of the critical layer mechanism as described in McKeon \& Sharma (2010). This critical layer mechanism gives rise to structures that are highly localized in the wall-normal direction. Considering the model eLNS, where the kinematic viscosity is augmented with an eddy viscosity profile, the large-scale structures that are modelled elongate in the wall-normal direction, and become coherent over a wider range of wall heights (figure 2). But even this model is not capable of capturing structures that show a high degree of coherence in the region close to the wall, as in DNS (figure 2(e)). The computation of coherence height also shows that the coherence of the large-scale structures from eLNS agrees with DNS, except in the region near the wall (figure 3). Further, the LCS obtained from eLNS scales with wall height (figure 4d). This shows that eLNS captures the self-similarity of the large-scale structures observed in experiments (Baars et al. 2017).

\section{Spectral Linear Stochastic Estimation}

Having looked at the coherence of the large scales from the linear models, we now look at the estimation of these structures using them. If a structure is coherent between two locations considered, we can expect to obtain an estimate of its statistics at one of the locations, based on a measurement at the other location.

\subsection{Description of SLSE}

The estimation tool used here is Spectral Linear Stochastic Estimation (SLSE), which was introduced in Tinney et al. (2006). Before showing any results, we first review SLSE. 

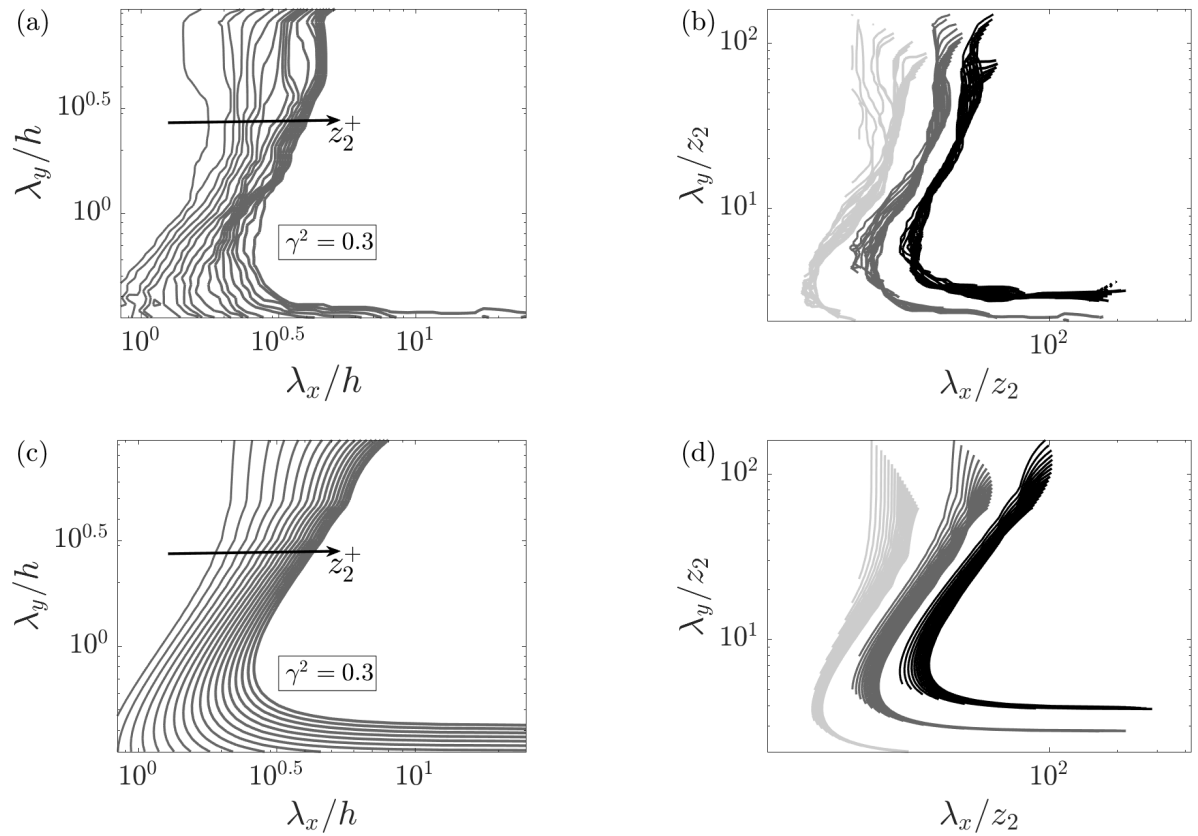

Figure 4: The contour level corresponding to $\gamma^{2}=0.3$ plotted for a fixed value of $z_{1}^{+} \approx 40$ and for different values of $z_{2}$ such that $2.6 \sqrt{R e_{\tau}} \leqslant z_{2}^{+} \leqslant 0.15 R e_{\tau}$. The contours are plotted $(\mathbf{a}, \mathbf{b})$ from the DNS dataset and $(\mathbf{c}, \mathbf{d})$ from eLNS. The plots in $(\mathbf{a}, \mathbf{c})$ show the contour levels plotted as a function of $\lambda_{x} / h$ and $\lambda_{y} / h$ while the plots in (b,d) show the collapse of the contour levels when plotted as a function of $\lambda_{x} / z_{2}$ and $\lambda_{y} / z_{2}$. (b,d) also show the collapse of the contours corresponding to $\gamma^{2}=0.1$ and $\gamma^{2}=0.5$, with darker colours corresponding to higher values of $\gamma^{2}$.

In SLSE, a complex-valued linear transfer kernel $H_{L}\left(z_{1}, z_{2} ; k_{x}, k_{y}\right)$ is defined that takes as input the Fourier coefficient of the streamwise velocity at a wavenumber pair $\left(k_{x}, k_{y}\right)$ and a wall-height $z_{2}\left(\hat{u}\left(z_{2} ; k_{x}, k_{y}\right)\right)$. The same quantity is estimated at a different wall-height $z_{1}\left(\hat{u}^{\prime}\left(z_{1} ; k_{x}, k_{y}\right)\right)$, and this can be written as

$$
\hat{u}^{\prime}\left(z_{1} ; k_{x}, k_{y}\right)=H_{L}\left(z_{1}, z_{2} ; k_{x}, k_{y}\right) \hat{u}\left(z_{2} ; k_{x}, k_{y}\right),
$$

where the ' represents the estimated quantity.

Multiplying (5.8) with the complex-conjugate of $\hat{u}\left(z_{2} ; k_{x}, k_{y}\right)$ and taking an ensemble average gives the transfer kernel $H_{L}\left(z_{1}, z_{2} ; k_{x}, k_{y}\right)$

$$
H_{L}\left(z_{1}, z_{2} ; k_{x}, k_{y}\right)=\frac{\left\langle\hat{u}\left(z_{1} ; k_{x}, k_{y}\right) \hat{u}^{*}\left(z_{2} ; k_{x}, k_{y}\right)\right\rangle}{\left\langle\hat{u}\left(z_{2} ; k_{x}, k_{y}\right) \hat{u}^{*}\left(z_{2} ; k_{x}, k_{y}\right)\right\rangle}=\left|H_{L}\left(z_{1}, z_{2} ; k_{x}, k_{y}\right)\right| e^{i \psi\left(z_{1}, z_{2} ; k_{x}, k_{y}\right)} .
$$

Here $\psi\left(z_{1}, z_{2} ; k_{x}, k_{y}\right)$ represents the phase of the transfer kernel. The denominator in (5.9) is the 2-D energy spectrum at $z_{2}$ and the numerator is the complex-valued crossspectrum between $z_{2}$ and $z_{1}$. The magnitude of the transfer kernel $\left|H_{L}\left(z_{1}, z_{2} ; k_{x}, k_{y}\right)\right|$ can be computed from the absolute value of the cross-spectrum and the spectrum

$$
\left|H_{L}\left(z_{1}, z_{2} ; k_{x}, k_{y}\right)\right|=\frac{\left|\left\langle\hat{u}\left(z_{1} ; k_{x}, k_{y}\right) \hat{u}^{*}\left(z_{2} ; k_{x}, k_{y}\right)\right\rangle\right|}{\left|\left\langle\hat{u}\left(z_{2} ; k_{x}, k_{y}\right) \hat{u}^{*}\left(z_{2} ; k_{x}, k_{y}\right)\right\rangle\right|}=\sqrt{\gamma^{2} \frac{\left\langle\left|\hat{u}\left(z_{1} ; k_{x}, k_{y}\right)\right|^{2}\right\rangle}{\left\langle\left|\hat{u}\left(z_{2} ; k_{x}, k_{y}\right)\right|^{2}\right\rangle}} .
$$




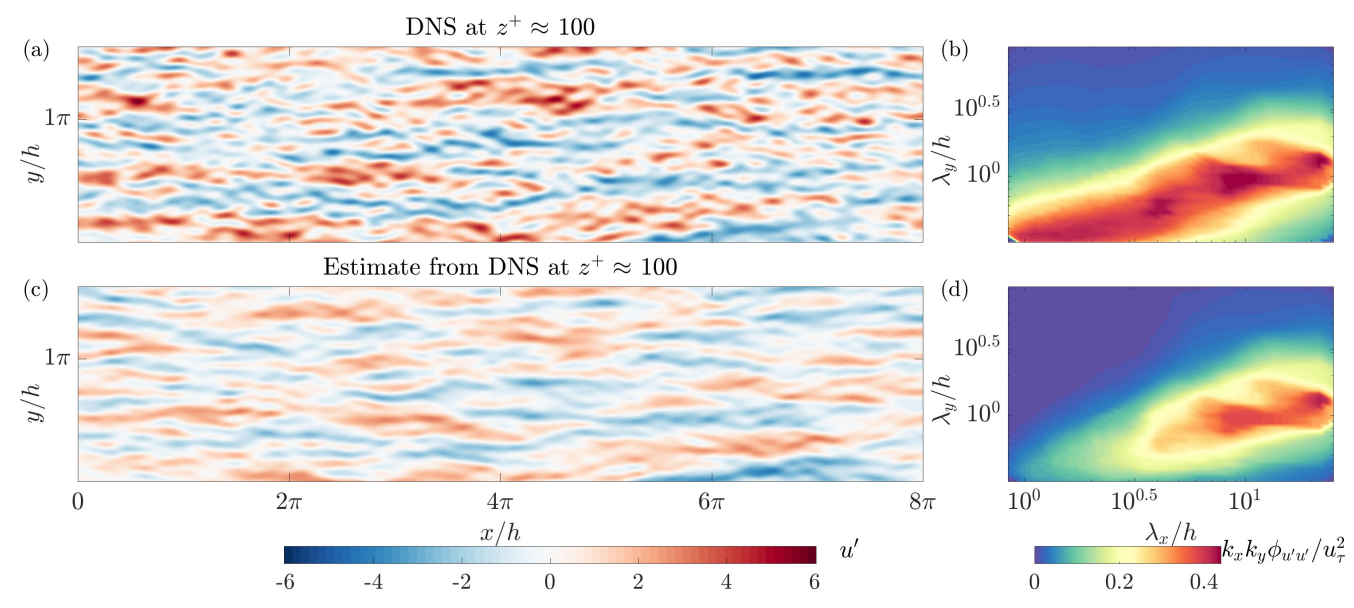

Figure 5: (a) The instantaneous streamwise velocity field and (b) the corresponding 2-D energy spectrum from the DNS dataset at $z_{1}^{+} \approx 100$. (c) The instantaneous streamwise velocity field estimated at $z_{1}^{+} \approx 100$ using the DNS dataset and $(\mathrm{d})$ the corresponding 2 -D energy spectrum. Here $z_{2}^{+} \approx 300$.

The magnitude of the transfer kernel can be understood as the linear coherence spectrum scaled by the ratio of the 2-D energy spectra at $z_{1}$ and $z_{2}$ (Baars et al. 2016).

Only the scales that are coherent between $z_{1}$ and $z_{2}$ can be properly estimated using SLSE. In other words, if a threshold value $\gamma_{T}^{2}$ is defined such that only the scales with $\gamma^{2}>\gamma_{T}^{2}$ are considered coherent, the transfer kernel $H_{L}\left(z_{1}, z_{2} ; k_{x}, k_{y}\right)$ can provide correct estimates only for these coherent scales. However, $H_{L}\left(z_{1}, z_{2} ; k_{x}, k_{y}\right)$ can have non-zero magnitudes at the incoherent scales with $\gamma^{2}<\gamma_{T}^{2}$. Hence, using $H_{L}\left(z_{1}, z_{2} ; k_{x}, k_{y}\right)$, we will erroneously obtain estimates for these incoherent scales. To avoid this, $H_{L}\left(z_{1}, z_{2} ; k_{x}, k_{y}\right)$ is set to zero for wavenumber pairs where $\gamma^{2}<\gamma_{T}^{2}$, yielding a filtered transfer kernel $H_{L}\left(z_{1}, z_{2} ; k_{x}, k_{y}\right)_{\text {filt }}$ (Tinney et al. 2006; Baars et al. 2016), which is used for estimation. Here, a threshold value of $\gamma_{T}^{2}=0.05$ is chosen. Provided $\gamma_{T}^{2}$ is kept sufficiently low, the exact choice of the threshold value does not have a significant effect on the results.

\subsection{Estimation of the 2-D energy spectrum}

Using SLSE, the 2-D energy spectrum at a wall-height $z_{1}$ can be estimated using only the 2-D energy spectrum at another wall-height $z_{2}$ as an input. In other words, the estimates of the 2-D energy spectrum can be obtained without directly estimating the time-resolved instantaneous velocity fields. From (5.8) we see that the estimation of the 2-D energy spectrum requires the magnitude of $H_{L}\left(z_{1}, z_{2} ; k_{x}, k_{y}\right)$ and can be written as

$$
\phi_{u u}^{\prime}\left(z_{1} ; k_{x}, k_{y}\right)=\left|H_{L}\left(z_{1}, z_{2} ; k_{x}, k_{y}\right)\right|^{2} \phi_{u u}\left(z_{2} ; k_{x}, k_{y}\right),
$$

where $\phi_{u u}\left(z ; k_{x}, k_{y}\right)$ represents the 2 -D energy spectrum of streamwise velocity at a wall height $z$.

\subsection{Estimation using the DNS dataset}

As an example, we consider the estimation of the velocity fluctuations and the 2-D energy spectrum at $z_{1}^{+} \approx 100$, taking the same quantities at $z_{2}^{+} \approx 300$ as an input. Before looking at the estimation from the linear models LNS and eLNS, we require a benchmark against which the estimates from the models can later be compared. 

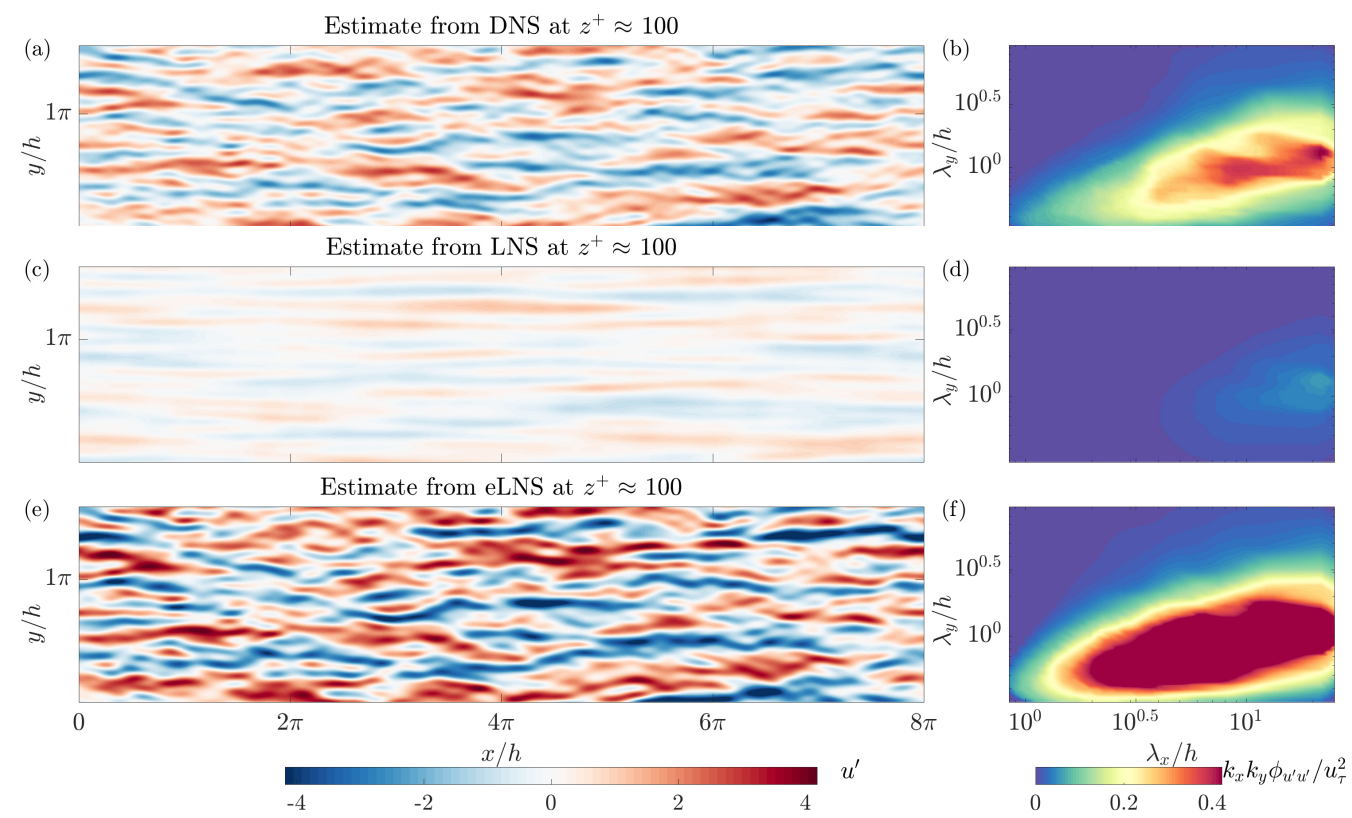

Figure 6: (a,c,e) The estimated instantaneous streamwise velocity field and (b,d,f) the corresponding 2-D energy spectrum obtained using (a,b) the DNS dataset, $(\mathrm{c}, \mathrm{d})$ LNS and $(\mathrm{e}, \mathrm{f})$ eLNS. The estimate is obtained at $z_{1}^{+} \approx 100$ based on a measurement at $z_{2}^{+} \approx 300$.

The best estimate using SLSE is that obtained by the transfer kernel $H_{L}\left(z_{1}, z_{2} ; k_{x}, k_{y}\right)$ computed from DNS (Baars et al. 2016). The velocity signals from DNS are hence used to obtain the correlations required to compute $H_{L}\left(z_{1}, z_{2} ; k_{x}, k_{y}\right)$ using (5.9), and the LCS computed from the same data (in §4.1) is used to filter the transfer kernel and obtain $\left(H_{L}\left(z_{1}, z_{2} ; k_{x}, k_{y}\right)\right)_{\text {filt }}$. Using this transfer kernel, the estimated velocity field at $z_{1}$ can be obtained with the velocity field at $z_{2}$ provided as an input. This is the 2-D equivalent of the estimation done in Baars et al. (2016) for the 1-D velocity field.

Figure $5(\mathrm{c})$ shows the estimate of the instantaneous velocity field at $z_{1}^{+} \approx 100$ obtained using the transfer kernel built from the DNS data. The corresponding 2-D energy spectrum is shown, in pre-multiplied form, in figure 5(d). According to (5.10) and (5.11), the energy spectrum of the estimated field at $z_{1}$ is simply the 2-D energy spectrum at $z_{1}$ multiplied by the LCS between $z_{1}$ and $z_{2}\left(\gamma^{2} \phi_{u u}\left(z_{1} ; k_{x}, k_{y}\right)\right)$. For comparison, the instantaneous velocity field and 2 -D energy spectrum at $z_{1}^{+} \approx 100$, directly obtained from the DNS dataset, is shown in figures 5(a) and 5(b) respectively. We see that only the larger scales remain in the estimated velocity field and 2-D energy spectrum at $z_{1}$. This is because only these scales are coherent between $z_{1}$ and $z_{2}$, as observed in $\S 4.1$.

\subsection{Estimation using the linear models}

The linear models can be used in conjunction with SLSE to estimate the instantaneous velocity field and the 2-D energy spectrum. The statistically converged velocity correlations from LNS and eLNS are required to compute $H_{L}\left(z_{1}, z_{2} ; k_{x}, k_{y}\right)$ for each model. These are obtained by solving the Lyapunov equation (2.5). Thereafter, the LCS computed from the models in $\S 4.1$ is used to obtain $H_{L}\left(z_{1}, z_{2} ; k_{x}, k_{y}\right)_{\text {filt. }}$. This $H_{L}\left(z_{1}, z_{2} ; k_{x}, k_{y}\right)_{\text {filt }}$ computed from the models is given as an input the velocity field 


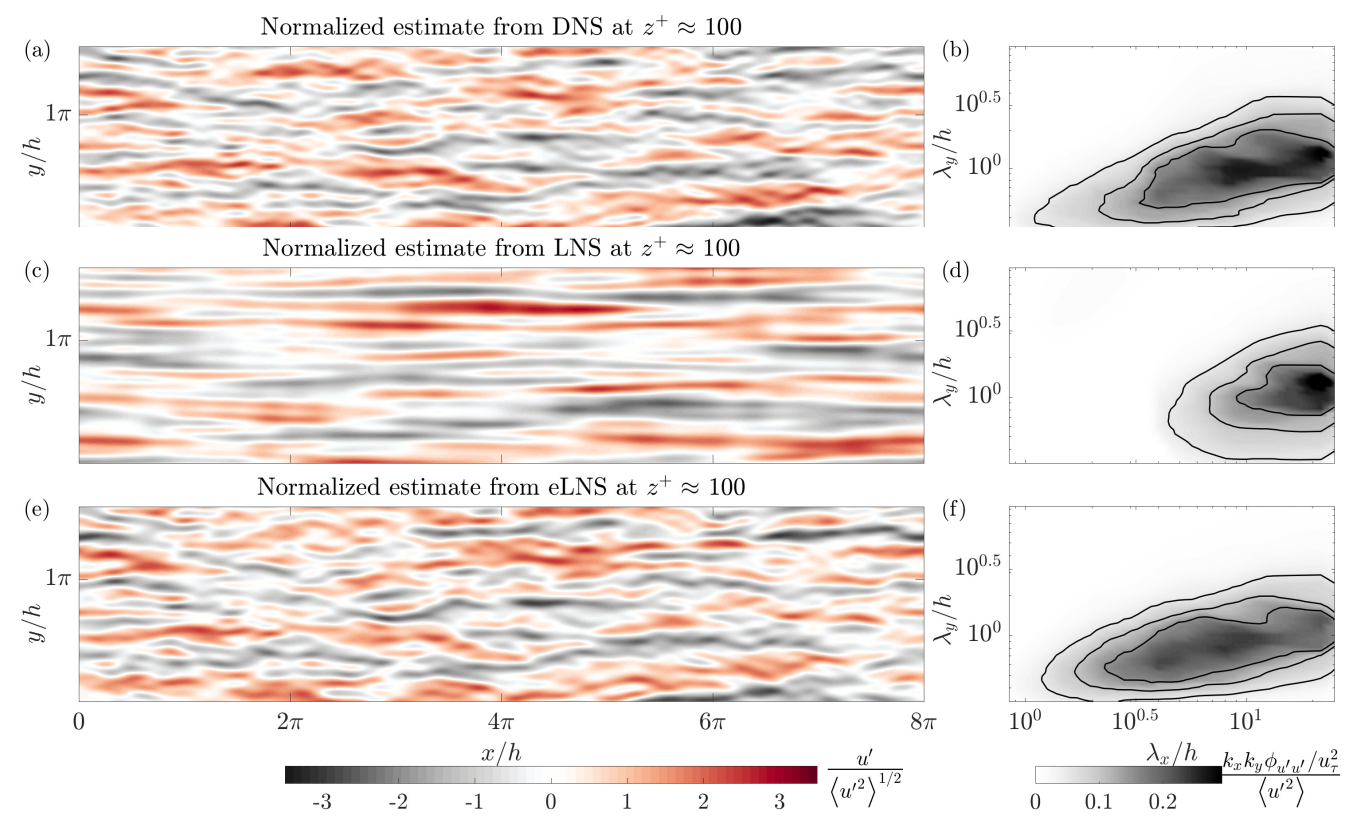

Figure 7: (a,c,e) The estimated instantaneous streamwise velocity field and (b,d,f) the corresponding 2-D energy spectrum obtained using $(\mathrm{a}, \mathrm{b})$ the DNS dataset, $(\mathrm{c}, \mathrm{d})$ LNS and (e,f) eLNS, normalized by $\left\langle u^{\prime 2}\right\rangle^{1 / 2}$ and $\left\langle u^{\prime 2}\right\rangle$ respectively. The estimate at $z_{1}^{+} \approx 100$ based on a measurement at $z_{2}^{+} \approx 300$ is shown. The contour lines in (b,d,f) correspond to $\left(k_{x} k_{y} \phi_{u^{\prime} u^{\prime}} / u_{\tau}^{2}\right) /\left\langle u^{\prime 2}\right\rangle=0.05,0.1$ and 0.15 .

or the 2-D energy spectrum (from DNS) at a measurement location of $z_{2}$. The estimated velocity field or $2-\mathrm{D}$ energy spectrum at $z_{1}$ is then obtained.

Figures $6(\mathrm{c})$ and $6(\mathrm{e})$ show the estimates of the instantaneous streamwise velocity field obtained at $z_{1}^{+} \approx 100$ using a measurement at $z_{2}^{+} \approx 300$, from LNS and eLNS, respectively. From these estimated velocity fields we see that the magnitude of the velocity fluctuations is not obtained correctly by either model. This is also reflected in the estimated 2-D energy spectra in figures $6(\mathrm{~d})$ and $6(\mathrm{f})$ where, for the combination of $z_{1}$ and $z_{2}$ considered, LNS underestimates the energy of the large scales while eLNS overestimates it. We observed in $\S 4.1$ that the large-scale structures modelled by LNS are coherent only over a narrow range of wall-heights. This observation explains the underestimation of energy by this model. On the other hand, eLNS overestimates the energy even though it better models the LCS, and hence the coherence of the large-scale structures. This is because the model does not correctly obtain the magnitude of the ratio of the spectra at $z_{1}$ and $z_{2}$, that is used in (5.10).

From figure $6(\mathrm{e})$ we see that, except for the actual magnitudes, the large-scale flow features modelled by eLNS are similar to DNS. Qualitatively, we see that eLNS captures the distribution of the large scale structures reasonably well, and therefore also approximately obtains the phase of these structures. To clarify this argument further, we normalize the estimated streamwise velocity and the energy spectra by $\left\langle{u^{\prime}}^{2}\right\rangle^{1 / 2}$ and $\left\langle{u^{\prime}}^{2}\right\rangle$ respectively. The normalization factor is computed separately for DNS, LNS and eLNS by integrating the estimated energy spectra. Figure 7 shows the normalized estimates corresponding to the estimates from DNS, LNS and eLNS in figure 6.

Considering the estimate from LNS in comparison to DNS, we see from figure 7 (c) that 
the model estimates only the very large scales, and only these scales remain energetic in the estimated 2-D energy spectrum shown in figure $7(\mathrm{~d})$. The estimate from LNS hence does not agree with those obtained from DNS. However, figure 7(e) shows that with the addition of an eddy viscosity profile, the distribution and phase of the large-scale structures are represented well by the linear model. From the estimated energy spectrum in figure $7(\mathrm{f})$ we see that the relative distribution of energy among the large-scale structures, i.e. the shape of the pre-multiplied energy spectrum, is captured reasonably well by the model.

\subsection{Varying the estimation location}

So far only one estimation location of $z_{1}^{+} \approx 100$ was considered. To investigate the quality of estimation over a range of wall-normal locations, we now consider multiple estimation locations in the inner-region of the flow at $10<z_{1}^{+}<200$, as shown in figure 8. In this figure the energy spectra are normalized by the variance, as previously shown in figure 7.

We first consider the estimates obtained using LNS. As $z_{1}$ moves away from $z_{2}$, the scales for which a non-zero estimate is obtained diminishes rapidly. An explanation for this can be obtained from the trends of the LCS plotted using LNS in $\S 4.1$, which showed that the coherence of the large scales quickly drops as $z_{1}$ moves away from $z_{2}$. In consequence, LNS does not estimate the shape of the 2 -D energy spectrum. (It should be noted that the energies estimated by LNS in figure $8(\mathrm{e})$ are very small due to the low values of coherence, and appears significant only due to the normalization.)

Now we look at the estimates obtained using eLNS. From figure 8 we see that this model provides a non-zero estimate for a wider range of $z_{1}$. This is consistent with the conclusion made using the LCS in $\S 4.1$ that the large-scale structures from eLNS are coherent over large wall-normal distances. Interestingly, for a range of $z_{1}$, the shape of the 2-D pre-multiplied energy spectrum is approximately estimated by eLNS. In other words, the model captures the relative distribution of energy among the large scales reasonably well when compared with DNS. The correspondence with DNS deteriorates as $z_{1}$ moves close to the wall. This is the region where the coherence from the model was observed to be too low in comparison to DNS in $\S 4.1$. Hence, though eLNS cannot estimate the magnitude of the 2-D energy spectrum, it can provide a reasonable estimate for the shape of the energy spectrum if $z_{1}$ is away from the wall.

\section{Conclusions}

In this study we computed the 2-D Linear Coherence Spectrum (LCS) for a turbulent channel flow at $R e_{\tau}=2000$, and compared it with the 2-D LCS computed from the linearized Navier-Stokes equations. The 2-D LCS computed from DNS data indicates the presence of large-scale structures that i) are coherent over large wall-normal distances (figure 2); ii) show high coherence close to the wall (figure 2(e) \& figure 3(b)); and iii) are self-similar (figure $4 \mathrm{~b}$ ). These observations are all consistent with those made using the 1-D LCS in Baars et al. (2017). We studied the extent to which each of these three features of the large-scale structures are captured by the linearized Navier-Stokes equations subject to stochastic forcing.

The stochastically forced linearized Navier-Stokes equations, denoted here as LNS, model structures that are highly localised in the wall-normal direction and are therefore coherent only over small wall-normal distances. By considering a model where the kinematic viscosity is augmented with an eddy viscosity profile, denoted here as eLNS, the 

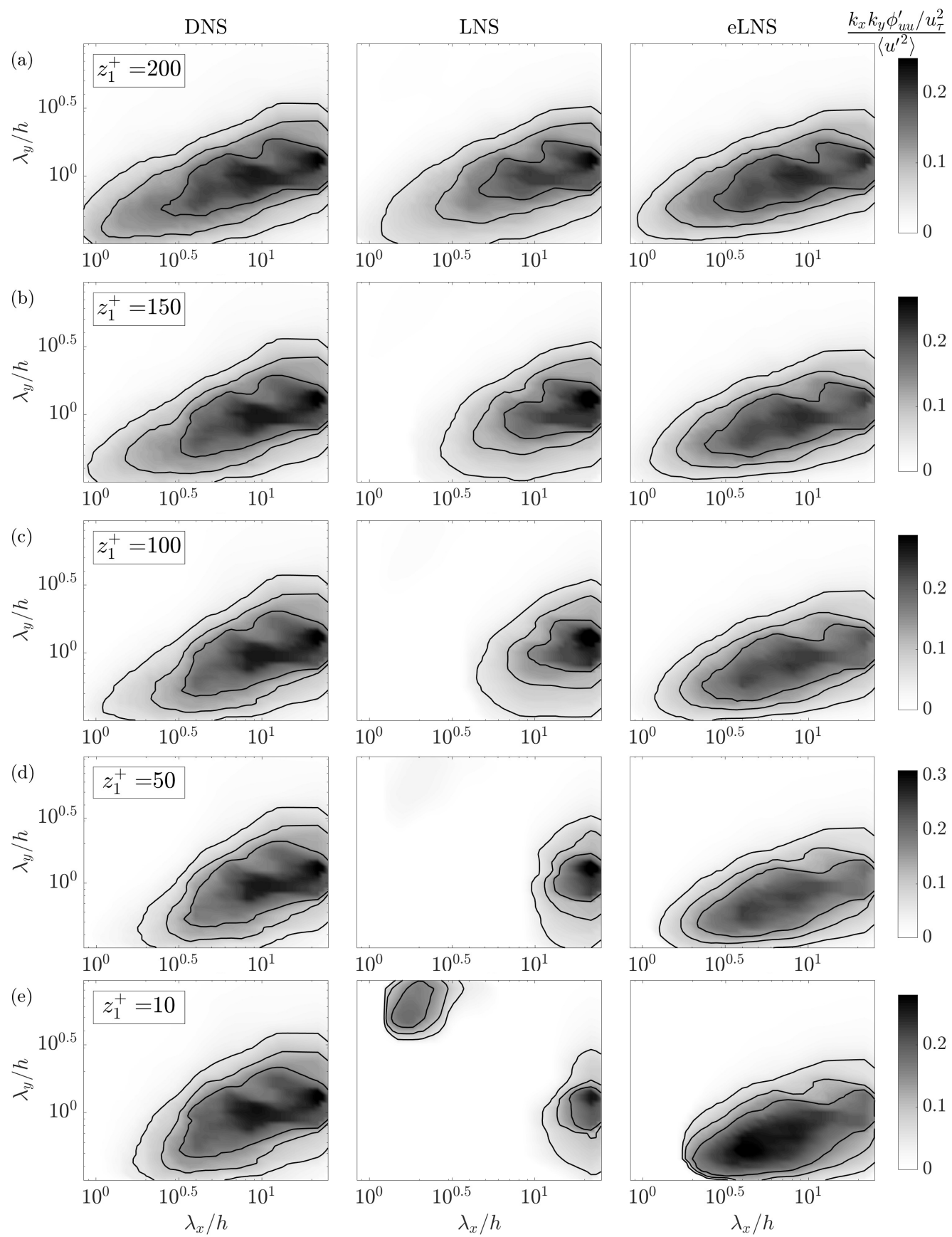

Figure 8: The estimates (in pre-multiplied form) of the 2-D energy spectrum normalized by $\left\langle u^{\prime 2}\right\rangle$, from DNS, LNS and eLNS, at $z_{1}^{+} \approx 200,150,100,50$ and 10 (top row to bottom row), with $z_{2}^{+} \approx 300$. The contour lines correspond to $\left(k_{x} k_{y} \phi_{u^{\prime} u^{\prime}} / u_{\tau}^{2}\right) /\left\langle u^{\prime 2}\right\rangle=$ $0.05,0.1$ and 0.15 . The regions where the estimate is zero (white) indicate scales that are incoherent and hence not estimated by the models. 
structures that are modelled become coherent over larger wall-normal distances and show better agreement with DNS (figure $2 \&$ figure $3(\mathrm{a})$ ). This suggests that eLNS captures the first of the three features, i.e. coherence over large wall-normal distances. However, the structures from eLNS show lower coherence than DNS in the near-wall region (figure 2 (e) \& figure 3(b)), and, therefore, eLNS does not capture the second of the three features considered, i.e. high coherence close to the wall. As well as modelling coherent large-scale structures, eLNS is also able to capture the third feature of the self-similarity of the structures, as observed from the wall scaling of the LCS (figure 4d).

These three features of the large-scale structures, as well as being interesting in their own right, also have an important effect on any efforts to estimate them, as seen in $\S 5$. In particular we used the linearized Navier-Stokes equations together with spectral linear stochastic estimation (SLSE) to build two linear estimators: the first using LNS and the second using eLNS. Each estimator uses the instantaneous velocity fluctuations or the 2-D energy spectrum at a measurement location of $z^{+} \approx 300$ (obtained from DNS) to estimate the same quantity over a range of estimation locations. For LNS the energy of the estimated structures quickly drops to zero as the estimation location moves away from the measurement location (figure 8). This is explained by the highly localized nature of the structures in the wall-normal direction (figure 2). For eLNS, meanwhile, the estimate remains energetic over a wider range of wall heights. This is consistent with the observations made using the LCS from eLNS (figure 2). Furthermore the model is able to capture the relative distribution of the large-scale structures and their energies across wavelengths (figure 7 and figure 8). However, there are two aspects of the streamwise velocity fields that cannot be captured by eLNS. First, the magnitude of the velocity fluctuations and hence the 2-D energy spectrum are not well-captured by the model. This is because, when considering a coherent large-scale eddy, the ratio of its energy between any two wall-normal locations is not well-captured by eLNS. And second, eLNS does not correctly capture the features of the flow in the near-wall region. Nevertheless the stochastically forced linearized Navier-Stokes equations, with the inclusion of an eddy viscosity profile, are able to model with reasonable accuracy the large-scale, self-similar structures observed in turbulent channel flows. This is encouraging for future efforts towards their modelling, estimation and control.

\section{Acknowledgements}

The authors are grateful for the financial support of the Australian Research Council. We would also like to thank the Fluid Dynamics Group of the Polytechnic University of Madrid (UPM) for the DNS dataset of Encinar et al. (2018) and Dr. Woutijn J. Baars for helpful discussions regarding this work.

\section{A. The Orr-Sommerfeld Squire form of LNS and eLNS}

The matrices $\boldsymbol{A}, \boldsymbol{B}$ and $\boldsymbol{C}$ in the Orr-Sommerfeld Squire form for LNS and eLNS in (2.4) are

$$
\begin{gathered}
\boldsymbol{A}\left(k_{x}, k_{y}\right)=\left[\begin{array}{cc}
\Delta^{-1} & 0 \\
0 & I
\end{array}\right]\left[\begin{array}{cc}
\mathcal{L}_{\mathcal{O S}} & 0 \\
-i k_{y} U^{\prime} & \mathcal{L}_{\mathcal{S} \mathcal{Q}}
\end{array}\right], \\
\boldsymbol{B}\left(k_{x}, k_{y}\right)=\left[\begin{array}{cc}
\Delta^{-1} & 0 \\
0 & I
\end{array}\right]\left[\begin{array}{ccc}
-i k_{x} \mathcal{D} & -i k_{y} \mathcal{D} & -k^{2} \\
i k_{y} & -i k_{x} & 0
\end{array}\right],
\end{gathered}
$$




$$
\boldsymbol{C}\left(k_{x}, k_{y}\right)=\frac{1}{k^{2}}\left[\begin{array}{cc}
i k_{x} \mathcal{D} & -i k_{y} \\
i k_{y} \mathcal{D} & i k_{x} \\
k^{2} & 0
\end{array}\right] .
$$

Here $\mathcal{D}$ and ' represent differentiation in the wall-normal direction, and $\Delta=\mathcal{D}^{2}-k^{2}$ where $k^{2}=k_{x}^{2}+k_{y}^{2}$. The matrices $\mathcal{L}_{O S}$ and $\mathcal{L}_{S Q}$ in (A.12) are the Orr-Sommerfeld and Squire operators respectively. For LNS they are

$$
\begin{aligned}
& \mathcal{L}_{\mathcal{O S}}=-i k_{x} U \Delta+i k_{x} U^{\prime \prime}+\left(1 / R e_{\tau}\right) \Delta^{2}, \\
& \mathcal{L}_{\mathcal{S} \mathcal{Q}}=-i k_{x} U+\left(1 / R e_{\tau}\right) \Delta .
\end{aligned}
$$

For eLNS they are

$$
\begin{aligned}
& \mathcal{L}_{\mathcal{O S}}=-i k_{x} U \Delta+i k_{x} U^{\prime \prime}+\nu_{T} \Delta^{2}+2 \nu_{T}^{\prime} \mathcal{D} \Delta+\nu_{T}^{\prime \prime}\left(\mathcal{D}^{2}+k^{2}\right) \\
& \mathcal{L}_{\mathcal{S Q}}=-i k_{x} U+\nu_{T} \Delta+\nu_{T}^{\prime} \mathcal{D}
\end{aligned}
$$

\section{REFERENCES}

Adrian, R. J. 1979 Conditional eddies in isotropic turbulence. Phys. Fluids 22 (11), 2065-2070.

Adrian, R. J., Meinhart, C.D \& Tomkins, C. D. 2000 Vortex organization in the outer region of the turbulent boundary layer. J. Fluid Mech. 422, 1-54.

Adrian, R. J. \& Moin, P. 1988 Stochastic estimation of organized turbulent structure: Homogeneous shear flow. J. Fluid Mech. 190, 531-559.

BaArs, W. J., Hutchins, N. \& Marusic, I. 2016 Spectral stochastic estimation of highReynolds-number wall-bounded turbulence for a refined inner-outer interaction model. Phys. Rev. Fluids 1, 054406.

BaArs, W. J., Hutchins, N. \& Marusic, I. 2017 Self-similarity of wall-attached turbulence in boundary layers. J. Fluid Mech. 823, R2.

BAlakumar, B. J. \& Adrian, R. J. 2007 Large- and very-large-scale motions in channel and boundary-layer flows. Phil. Trans. R. Soc. Lond. 365 (1852), 665-681.

Bonnet, J. P., Delville, J., Glauser, M. N., Antonia, R. A., Bisset, D. K., Cole, D. R., Fiedler, H. E., Garem, J. H., Hilberg, D., Jeong, J., Kevlahan, N. K. R., Ukeiley, L. S. \& Vincendeau, E. 1998 Collaborative testing of eddy structure identification methods in free turbulent shear flows. Exp. Fluids 25, 197-225.

Brown, G. L. \& Thomas, A. S. W. 1977 Large structure in a turbulent boundary layer. Phys. Fluids 20 (10), S243-S252.

Butler, K. M. \& FARrell, B. F. 1993 Optimal perturbations and streak spacing in wallbounded turbulent shear flow. Phys. Fluids A 5 (3), 774-777.

CEss, R. D. 1958 A survey of the literature on heat transfer in turbulent tube flow. Res. Rep pp. 8-0529.

Cole, D. R. \& Glauser, M. N. 1998 Applications of stochastic estimation in the axisymmetric sudden expansion. Phys. Fluids 10 (11), 2941-2949.

Cossu, C., Pujals, G. \& Depardon, S. 2009 Optimal transient growth and very large-scale structures in turbulent boundary layers. J. Fluid Mech. 619, 79-94.

Del Álamo, J. C. \& Jiménez, J. 2006 Linear energy amplification in turbulent channels. J. Fluid Mech. 559, 205-213.

Dennis, D. J. C. \& Nickels, T. B. 2011 Experimental measurement of large-scale threedimensional structures in a turbulent boundary layer. part 1. vortex packets. J. Fluid Mech. 673, 180-217.

Encinar, M. P., Vela-Martín, A., García-Gutiérrez, A. \& Jiménez, J. 2018 A secondorder consistent, low-storage method for time-resolved channel flow simulations. ArXiv e-prints, arXiv: 1808.06461.

Ewing, D. \& Citriniti, J. H. 1999 Examination of a LSE/POD complementary technique using single and multi-time information in the axisymmetric shear layer. In IUTAM Symposium on simulation and identification of organized structures in flows, pp. 375-384. Springer.

FArrell, B. F. \& IoAnnou, P. J. 1993 Optimal excitation of three-dimensional perturbations in viscous constant shear flow. Phys. Fluids A 5 (6), 1390-1400. 
FARrell, B. F. \& IoAnnou, P. J. 1998 Perturbation structure and spectra in turbulent channel flow. Theor. Comput. Fluid Dyn. 11, 237-250.

Ganapathisubramani, B., Longmire, E. K. \& Marusic, I. 2003 Characteristics of vortex packets in turbulent boundary layers. J. Fluid Mech. 478, 35-46.

Guala, M., Hommema, S. E. \& Adrian, R. J. 2006 Large-scale and very-large-scale motions in turbulent pipe flow. J. Fluid Mech. 554, 521-542.

Hellström, L. H. O., Marusic, I. \& Smits, A. J. 2016 Self-similarity of the large-scale motions in turbulent pipe flow. J. Fluid Mech. $\mathbf{7 9 2}$.

Hoyas, S. \& JimÉnEZ, J. 2006 Scaling of the velocity fluctuations in turbulent channels up to $R_{\tau}=2003$. Phys. Fluids 18 (1), 011702.

Hutchins, N., Hambleton, W. T. \& Marusic, I. 2005 Inclined cross-stream stereo particle image velocimetry measurements in turbulent boundary layers. J. Fluid Mech. 541, 21-54.

Hutchins, N. \& MARusic, I. $2007 a$ Evidence of very long meandering features in the logarithmic region of turbulent boundary layers. J. Fluid Mech. 579, 1-28.

Hutchins, N. \& Marusic, I. $2007 b$ Large-scale influences in near-wall turbulence. Phil. Trans. R. Soc. Lond. 365 (1852), 647-664.

HWANG, Y. 2015 Statistical structure of self-sustaining attached eddies in turbulent channel flow. J. Fluid Mech. 767, 254-289.

Hwang, Y. \& Cossu, C. 2010 a Amplification of coherent streaks in the turbulent Couette flow: an input-output analysis at low Reynolds number. J. Fluid Mech. 643, 333-348.

Hwang, Y. \& Cossu, C. $2010 b$ Linear non-normal energy amplification of harmonic and stochastic forcing in the turbulent channel flow. J. Fluid Mech. 664, 51-73.

Illingworth, S. J., Monty, J. P. \& Marusic, I. 2018 Estimating large-scale structures in wall turbulence using linear models. J. Fluid Mech. 842, 146-162.

JimÉnez, J. 2012 Cascades in wall-bounded turbulence. Annu. Rev. Fluid Mech. 44.

Jiménez, J., Del Álamo, J. C. \& Flores, O. 2004 The large-scale dynamics of near-wall turbulence. J. Fluid Mech. 505, 179-199.

Kim, K. C. \& Adrian, R. J. 1999 Very large-scale motion in the outer layer. Phys. Fluids $11(2), 417-422$.

Klewicki, J., Fife, P. \& Wei, T. 2009 On the logarithmic mean profile. J. Fluid Mech. 638, 73-93.

Lozano-Durán, A., Flores, O. \& Jiménez, J. 2012 The three-dimensional structure of momentum transfer in turbulent channels. J. Fluid Mech. 694, 100-130.

Marusic, I. 2001 On the role of large-scale structures in wall turbulence. Phys. Fluids 13 (3), $735-743$.

Marusic, I. \& Monty, J. P. 2019 Attached eddy model of wall turbulence. Annu. Rev. Fluid Mech. 51.

Marusic, I., Monty, J. P., Hultmark, M. \& Smits, A. J. 2013 On the logarithmic region in wall turbulence. J. Fluid Mech. 716, R3.

McKeon, B. J. \& Sharma, A. S. 2010 A critical-layer framework for turbulent pipe flow. J. Fluid Mech. 658, 336-382.

McKeon, B. J., Sharma, A. S. \& Jacobi, I. 2013 Experimental manipulation of wall turbulence: a systems approach. Phys. Fluids 25 (3), 031301.

Moarref, R., Jovanović, M. R., Tropp, J. A., Sharma, A. S. \& McKeon, B. J. 2014 A low-order decomposition of turbulent channel flow via resolvent analysis and convex optimization. Phys. Fluids 26 (5), 051701.

Modrref, R., Sharma, A. S., Tropp, J. A. \& McKeon, B. J. 2013 Model-based scaling of the streamwise energy density in high-reynolds number turbulent channels. J. Fluid Mech. 734, 275-316.

Monty, J. P., Stewart, J. A., Williams, R. C. \& Chong, M. S. 2007 Large-scale features in turbulent pipe and channel flows. J. Fluid Mech. 589, 147-156.

Pujals, G., García-Villalba, M., Cossu, C. \& Depardon, S. 2009 A note on optimal transient growth in turbulent channel flows. Phys. Fluids 21 (1), 015109.

Reynolds, W. C. \& Hussain, A. K. M. F. 1972 The mechanics of an organized wave in turbulent shear flow. Part 3. Theoretical models and comparisons with experiments. J. Fluid Mech. 54 (02), 263-288. 
Reynolds, W. C. \& Tiederman, W. G. 1967 Stability of turbulent channel flow, with application to malkus's theory. J. Fluid Mech. 27 (2), 253-272.

Schmid, P. J. 2007 Nonmodal stability theory. Annu. Rev. Fluid Mech. 39, 129-162.

Sharma, A. S. \& McKeon, B. J. 2013 On coherent structure in wall turbulence. J. Fluid Mech. 728, 196-238.

Smits, A. J., McKeon, B. J. \& Marusic, I. 2011 High-Reynolds number wall turbulence. Annu. Rev. Fluid Mech. 43, 353-375.

Tinney, C. E., Coiffet, F., Delville, J., Hall, A. M., Jordan, P. \& Glauser, M. N. 2006 On spectral linear stochastic estimation. Exp. Fluids 41 (5), 763-775.

Tomkins, C. D. \& Adrian, R. J. 2005 Energetic spanwise modes in the logarithmic layer of a turbulent boundary layer. J. Fluid Mech. 545, 141-162.

Townsend, A. A. 1976 The structure of turbulent shear flow. Cambridge university press.

Trefethen, L. N., Trefethen, A. E., Reddy, S. \& Driscoll, T. A. 1993 Hydrodynamic stability without eigenvalues. Science 261, 578-584.

Waleffe, F., Kim, J. \& Hamilton, J. M. 1993 On the origin of streaks in turbulent shear flows. In Turbulent Shear Flows 8, pp. 37-49. Springer.

Willis, A. P., Hwang, Y. \& Cossu, C. 2010 Optimally amplified large-scale streaks and drag reduction in turbulent pipe flow. Phys. Rev. E 82 (3), 036321.

Zhou, J., Adrian, R. J., Balachandar, S. \& Kendall, T. M. 1999 Mechanisms for generating coherent packets of hairpin vortices in channel flow. J. Fluid Mech. 387, 353396.

Zhou, K., Doyle, J. C. \& Glover, K. 1996 Robust and optimal control, , vol. 40. PrenticeHall. 